o teste, pois este apresentava histórico de administração exógena de corticóide, sugerindo-se um quadro de $\mathrm{HAC}$ iatrogênico. Atualmente, a ocorrência do $\mathrm{HAC}$ tem aumentado na clínica de pequenos animais, tornando-se essencial inclui-lo nos diagnósticos diferenciais de outras endocrinopatias, além de outras causas de poliúria e polidipsia, como doenças renais e hepáticas. Para isso, é importante o reconhecimento da doença e sua predisposição.

\title{
56 - Emprego do bezafibrato no tratamento da hipertrigliciridemia primária em Schnauzer. Relato de dois casos
}

1- Clínica Veterinária Alto da Lapa da Universidade de Santo Amaro, Universidade Bandeirantes e AnhembiMorumbi, São Paulo-SP

2- Clínica Veterinária Alto da Lapa, Pet Blue, São Paulo-SP

A hiperlipidemia é o termo usado para descrever a concentraçào excessiva de colesterol ou de triglicérides no soro ou plasma de animal em jejum. É o resultado direto do aumento na produçào de lipoproteínas ou da redução da degradação destas moléculas. $\mathrm{O}$ estado de hiperlipidemia é caracterizado como primário (ou familiar) ou secundário à doença endócrina metabólica. A hiperlipidemia por excesso de concentração de triglicérides é a forma grave de distúrbio no transporte lipídico em cães. A hipertrigliceridemia não apenas sinaliza a presença de distúrbio metabólico subjacente, mas é fator de risco importante para a saúde do paciente, $\mathrm{e}$, justifica a intervenção dietética $\mathrm{e} / \mathrm{ou}$ terapêutica. Os cães afetados estão sob o risco de desenvolver pancreatites, convulsões e distúrbios gastro intestinais. O mecanismo exato não foi elucidado, mas é provável que a diminuição da atividade da lipase lipoprotéica ou, ainda, a ausência da apoproteina C II de superfície estejam relacionadas ao acúmulo de triglicérides na circulaçào. Relatos prévios sugerem que animais da raça schnauzer adultos ou idosos estejam predispostos a hiperlipidemia primaria ou familiar, não associada a distúrbios endócrinos subjacentes. A restrição de gordura dos alimentos é a primeiro passo do tratamento, por meio da utilização da dieta com porcentagem de gordura menor que $10 \%$ da matéria seca. Como coadjuvante, nos casos em que a dieta não se mostra eficaz, pode-se utilizar as drogas hipolipidemiantes, a exemplo do bezafibrato, do grupo dos fibratos. Estes são capazes de diminuir os niveis séricos de colesterol, de triglicérides (notavelmente VLDL e LDL), além de reduzir as concentrações de fibrinogênio. Adicionalmente, propiciam a elevação dos níveis séricos de HDL. São eficazes na maioria dos tipos de dislipidemias, tanto primárias quanto secundárias. $\mathrm{O}$ mecanismo molecular na qual o bezafibrato reduz o depósito de gordura na célula não foi completamente elucidado. Sabe-se que ele é responsável pelo aumento na degradação de ácidos graxos, reduzindo a capacidade de sintetizar os triglicerideos. Nào existem relatos publicados sobre o uso desta droga em animais da espécie canina em toda a literatura compilada. Objetivou-se avaliar a eficácia do benzafibrato em càes que apresentavam aumento do nível de triglicérides plasmático e que não responderam à terapia nutricional instituída. No presente trabalho, realizado na Clínica Veterinária Alto da Lapa, foram selecionados dois animais da raça schnauzer, sendo um macho, oito anos, não castrado (cão 1) e, o outro, uma fêmea, 12 anos, não castrada (cão 2), que apresentaram hipertrigliceridemia mesmo após a utilização prolongada de dieta hipocalórica, com baixos niveis de gordura e altos teores de fibra. Os valores de triglicérides pré-terapia com o bezafibrato foram iguais a $891 \mathrm{mg} / \mathrm{dl}$ (cão 1) e $4398 \mathrm{mg} / \mathrm{dl}$ (cão 2). Foi administrado o bezafibrato na dose de $2,5 \mathrm{mg} / \mathrm{kg}$, a cada 12 horas, e após 90 dias do início do tratamento os valores de triglicérides foram iguais a $306,2 \mathrm{mg} / \mathrm{dl}$ (cão 1) e $419 \mathrm{mg} / \mathrm{dl}$ (cão 2). Ao longo do período, o uso do medicamento não resultou em efeitos deletérios ao perfil hematológico $\mathrm{e}$ às funções renal e hepática, como constado pelas realizações mensais de hemograma completo, além das determinações séricas de uréia e creatinina, de ALTe FA. Concluímos que, para o controle da hipertrigliceridemia primária em cães da raça schnauzer, o bezafibrato se mostrou eficaz e, aparentemente, sem efeitos colaterais. 